\title{
Análise de diferentes protocolos dos exercícios aeróbicos na dor em mulheres com
}

\section{fibromialgia: uma revisão sistemática}

\author{
Analysis of different protocols of aerobic exercises in pain in women with fibromyalgia: a \\ systematic review \\ Análisis de diferentes protocolos de ejercicio aeróbico en el dolor en mujeres con fibromialgia: una \\ revisión sistemática
}

Recebido: 19/02/2021 | Revisado: 28/02/2021 | Aceito: 03/03/2021 | Publicado: 09/03/2021

\author{
Claudia Kenia Fernandes da Silva \\ ORCID: https://orcid.org/0000-0001-5844-1499 \\ Faculdade Estácio de Natal, Brasil \\ E-mail: claudiakeni@ hotmail.com \\ Edivan Germano de Souza \\ ORCID: https://orcid.org/0000-0002-3174-2520 \\ Faculdade Estácio de Natal, Brasil \\ E-mail: ed_germano10@ hotmail.com \\ Hawerton da Silva Barbosa \\ ORCID: https://orcid.org/0000-0003-2677-5181 \\ Faculdade Estácio de Natal, Brasil \\ E-mail: hawertonbarbosa@hotmail.com \\ José Felipe Costa da Silva \\ ORCID: https://orcid.org/0000-0001-5313-0683 \\ Universidade Federal do Rio Grande do Norte, Brasil \\ E-mail: felipedoshalom@yahoo.com.br
}

\begin{abstract}
Resumo
Introdução: A fibromialgia apresenta-se como uma condição clínica reumatológica que é tida como uma síndrome dolorosa crônica. Dentre os sintomas destaca-se a dor, fadiga muscular, dificuldades para dormir, rigidez matinal e distúrbios cognitivos. Os exercícios podem auxiliar esses indivíduos na preservação da aptidão física e aumento da endorfina que juntos irão proporcionar uma melhora nana qualidade de vidas desses pacientes. Objetivos: avaliar os tipos de exercícios aeróbicos, em relação à frequência, intensidade e duração e os benefícios na redução do limiar da dor na fibromialgia. Métodos: o presente estudo trata-se de uma revisão sistemática. Onde as buscas foram realizadas através das bases de dados SciELO, PubMed, PEDro, Periódicos CAPES, LILACS e busca ativa das referências. Foram selecionados estudos que estivessem relacionados ao público feminino, ensaios clínicos, e estudos que tenham a dor como desfecho primário. Nos idiomas inglês e português. Resultados: Os estudos relacionados ao exercício aeróbico em pacientes com FM demostraram benefícios na redução da dor. Os mesmos evidenciaram que os pacientes que utilizaram o exercício aeróbico em seus protocolos contemplaram ganhos significativos na redução da sintomatologia dolorosa da FM. Conclusão: Sugere-se que o exercício aeróbico como tratamento não medicamentoso colabora com a redução da sintomatologia dolorosa em pacientes fibromiálgicos, desde que respeitem os limites de intensidade, frequências e duração desses indivíduos.
\end{abstract}

Palavras-chave: Fibromialgia; Dor; Exercícios aeróbicos; Protocolos; Fisioterapia.

\begin{abstract}
Introduction: Fibromyalgia presents itself as a clinical rheumatological condition that is considered a chronic painful syndrome. Among the symptoms, pain, muscle fatigue, difficulty sleeping, morning stiffness and cognitive disorders stand out. Exercises can assist these individuals in preserving physical fitness and increasing endorphins, which together will provide an improvement in the quality of life of these patients. Objectives: to evaluate the types of aerobic exercises, in relation to frequency, intensity and duration and the benefits in reducing the pain threshold in fibromyalgia. Methods: the present study is a systematic review. Where searches were performed using the SciELO, PubMed, PEDro, CAPES, LILACS databases and active search of references. Studies were selected that were related to the female audience, clinical trials, and studies that have pain as the primary outcome. In English and Portuguese. Results: Studies related to aerobic exercise in patients with FM have shown benefits in reducing pain. They showed that patients who used aerobic exercise in their protocols contemplated significant gains in reducing the painful symptoms of FM. Conclusion: It is suggested that aerobic exercise as a non-medication treatment contributes to the
\end{abstract}


reduction of painful symptoms in fibromyalgia patients, as long as they respect the limits of intensity, frequencies and duration of these individuals.

Keywords: Fibromyalgia; Ache; Aerobic exercises; Protocols; Physiotherapy.

\section{Resumen}

Introducción: La fibromialgia se presenta como una condición clínica reumatológica que se considera un síndrome doloroso crónico. Entre los síntomas destacan el dolor, la fatiga muscular, las dificultades para dormir, la rigidez matutina y los trastornos cognitivos. Los ejercicios pueden ayudar a estas personas a preservar la aptitud física y aumentar las endorfinas, lo que en conjunto proporcionará una mejora en la calidad de vida de estos pacientes. Objetivos: evaluar los tipos de ejercicios aeróbicos, en relación a la frecuencia, intensidad y duración y los beneficios en la reducción del umbral de dolor en la fibromialgia. Métodos: el presente estudio es una revisión sistemática. Donde se realizaron búsquedas utilizando las bases de datos SciELO, PubMed, PEDro, CAPES, LILACS y búsqueda activa de referencias. Se seleccionaron estudios relacionados con el público femenino, ensayos clínicos y estudios que tienen el dolor como resultado principal. En inglés y portugués. Resultados: Los estudios relacionados con el ejercicio aeróbico en pacientes con FM han demostrado beneficios en la reducción del dolor. Demostraron que los pacientes que usaron ejercicio aeróbico en sus protocolos contemplaron ganancias significativas en la reducción de los síntomas dolorosos de la FM. Conclusión: Se sugiere que el ejercicio aeróbico como tratamiento no farmacológico contribuye a la reducción de los síntomas dolorosos en pacientes con fibromialgia, siempre que respeten los límites de intensidad, frecuencias y duración de estos individuos.

Palabras clave: Fibromialgia; Dolor; Ejercicios aeróbicos; Protocolos; Fisioterapia.

\section{Introdução}

A fibromialgia (FM) apresenta-se como uma condição clínica reumatológica de etiologia desconhecida, sua principal característica é a dor difusa pelo corpo que acomete o sistema musculoesquelético (Martins et al., 2014). A FM é tida como uma síndrome dolorosa crônica que tem sua patologia complexa, multifatorial e ainda não completamente esclarecida. Dentre os sintomas da FM destaca-se a dor, fadiga muscular, dificuldades para dormir, rigidez matinal e distúrbios cognitivos. Consequentemente associa-se a outras comorbidades, que contribuem com o sofrimento e piora da qualidade de vida destes pacientes, impossibilitando-os nas diversas atividades de vida diária (Heymann et al., 2010).

Um estudo de revisão literária (Marques et al., 2017) apontou que os valores da prevalência da FM na população mundial varia entre 0,2 e $6,6 \%$, nas mulheres 2,4 e 6,8\%, nas áreas urbanas 0,7 e $11,4 \%$, e nas áreas rurais 0,1 e $5,2 \%$. No Brasil, Senna et al (2004) determinou que a prevalência de fibromialgia é de 2,5\% da população, apresentando em sua maioria no sexo feminino, com uma relação de (9:1). Em relação à idade, 40,8\% dos pacientes de fibromialgia têm entre 35 e 44 anos.

Acredita-se que a fisiopatologia da FM envolve o desequilíbrio no organismo entre a percepção dolorosa e os mecanismos de modulação das vias descendentes inibitórias da dor. Esse conjunto de estruturas é denominado sistema inibidor de dor e os principais neurotransmissores envolvidos no seu funcionamento são a serotonina e noradrenalina ao nível do tronco encefálico que influencia de maneira direta na percepção dolorosa e as dinorfinas e encefalinas a nível segmentar medular. No entanto, ficou constatado que inicialmente os estímulos dolorosos seriam capazes de efetuar uma sensação desagradável da dor, e que posteriormente estimulariam estas vias descendentes, concretizando assim como moduladores da dor (Lorena et al., 2016; Riberto et al., 2004).

Os distúrbios neuroendócrinos, como alterações nos níveis de cortisol e/ou de melatonina, parecem estar envolvidos na fisiopatologia da FM (Carvalho et al., 2008; Pereira Pernambuco, 2014). Dentre essas hipóteses, se destacam as que apontam para níveis reduzidos de cortisol entre pacientes com FM (Carvalho et al., 2008). Essa alteração estaria relacionada a uma disfunção do eixo Hipotálamo - Pituitária - Adrenal (HPA), também conhecido como eixo de resposta ao estresse. Esse eixo neuroendócrino participa da manutenção da homeostase corporal, mas sempre que o organismo se depara com um agente estressor de qualquer natureza (físico ou psicoemocional), o eixo HPA entra em hiperatividade o que resulta em níveis elevados de liberação de cortisol (Crofford et al., 2004).

Por tanto a FM tem sido constantemente estudada por se tratar de uma condição reumática que envolve 
significativamente um quadro crônico de dor difusa. De acordo com os critérios do Colégio Americano de Reumatologia ACR (Wolfe et al., 1990), a dor da FM é generalizada, constante e está presente nos segmentos superior e inferior, bilateralmente. Segundo a diretriz para o diagnóstico da fibromialgia um dos critérios para avaliar a doença é baseado na presença de 11 dos 18 pontos dolorosos encontrados (tender points). Entretanto, em 2010 o ACR (Wolfe et al., 2010), elaborou novos critérios para classificar o diagnóstico da FM, pois aproximadamente $25 \%$ dos pacientes não satisfaziam os critérios de classificação da ACR de 1990 somente com a avaliação dos pontos dolorosos. Também, foi incluído os sintomas cognitivos, sono não reparador e fadiga, além de elaborar um Índice de Dor Generalizada, associado à Escala de Gravidade de Sintomas, onde ambos são baseados nos sintomas do paciente (Batista et al., 2016; Häuser et al., 2010; Wolfe et al., 2010).

Segundo a Diretriz para o manejo da dor sobre a FM o tratamento deve ser multidisciplinar, com adesão de tratamento farmacológico como: antidepressivos, inibidores de serotonina, analgésicos e medicamentos indutores do sono. E não farmacológicos como terapia cognitivo-comportamental, acupuntura, massagem terapêutica, balneoterapia, alongamentos, exercício de fortalecimento e exercício aeróbico (Fitzcharles et al., 2013).

Estudos de Marques et al (2002) apontam que o exercício físico tem diversos benefícios tais como: o aumento de serotonina, do hormônio do crescimento e da quantidade de mioglobina. Todos esses benefícios colaboram para diminuição dos sintomas da dor, melhora no sono, a fadiga, da ansiedade e de outros sintomas da fibromialgia. Os exercícios aeróbicos podem ser incluídos no tratamento de pacientes fibromiálgicos visando beneficiar o paciente promovendo a redução da dor, ganho de amplitude de movimento (ADM) e consequentemente ganho de força e aumento da capacidade funcional para os grupos musculares (Helfenstein et al., 2012).

Esse efeito terapêutico do exercício pode influenciar múltiplos aspectos da FM, como melhoria das alterações isquêmicas e metabólicas nos locais dos tender points, aumento do nível de endorfina, melhoria do estado mental e do padrão do sono. A prática de exercícios proporciona sensação de bem-estar e de autocontrole, apresentando efeito analgésico, relacionado às dores características da patologia (Marques et al., 2002; Sabbag et al., 2000). Ainda, estabelece uma postura adequada e auxilia no controle da massa corporal, da ansiedade e da condição cardiovascular (Ribeiro et al., 2005).

Matsutani et al (2012) utiliza-se de diversas modalidades de exercícios, incluindo a esteira Ergométrica, que segundo, utilizou com a intensidade sendo definida de acordo com a Frequência Cardíaca (FC) (Baptista et al., 2012). A dança é um tipo de exercício aeróbico que tem grande aceitação, contribuindo modalidade da dança zumba que faz a associação dos princípios básicos do treinamento físico aeróbico, segundo a literatura existem benefícios dessa modalidade em pacientes com FM(Assunção Júnior et al., 2018; Cruz et al., 2017; Ljubojević et al., 2014). A Hidroterapia foi utilizada como o recurso terapêutico no exercício aeróbico em pacientes de FM aproveitando os princípios físicos da água que é proporcionado ao indivíduo (Mannerkorpi, 2005; Mosmann et al., 2006). Pode-se utilizar a caminhada como exercício aeróbico variando de acordo com o nível de condicionamento físico de cada indivíduo para que haja um efeito sobre a Síndrome (Klumb et al., 2012).

Dentre os diferentes programas de EA, as atividades de baixa intensidade e de longa duração, têm sido destacadas como eficientes no manejo do paciente fibromiálgicos (Mandini et al., 2001). Contudo, os estudos apresentam diferentes protocolos de intervenção, que apontam modalidades distintas no qual apresentam frequência que segundo Cooper (1982), sugere que o treinamento aeróbico ocorra no mínimo 3 vezes por semana, mas se possível 4 vezes por semana. Entretanto a American College of Sports Medicine, (1998), recomenda uma frequência de treinamento de 3 a 5 dias por semana, constata-se que na duração a American College of Sports Medicine, (1998) American College of Sports Medicine, (1998), recomenda uma duração de 15 a 60 minutos contínuos. Mcardle (2016), afirma que o treino não precisa ultrapassar 30 minutos. Pollock (1993), descreve que o treino pode ser de 20 a 30 minutos. Entretanto, Consenza (2000), afirma que em seus estudos na literatura encontrou como um bom volume de atividade aeróbica uma faixa de 40 a 45 minutos contínuos. Outros autores (Boter et al., 
2020; del Vecchio et al., 2013), afirmam que a intensidade, a frequência semanal, a duração das sessões e o tipo de programa influenciam diretamente o efeito do treinamento aeróbico.

Como visto acima, ainda não foi determinado qual exercício é mais adequado, com relação à frequência, intensidade e duração eficaz (Clark et al., 2011). Neste contexto, surge a necessidade de um estudo que aponte os protocolos de exercícios aeróbicos que apresentem as melhores respostas clínicas na redução da sintomatologia dolorosa em pacientes com fibromialgia. E assim trazer benefícios para o tratamento da dor em pacientes acometidos pela fibromialgia, outrossim colaborar com toda sociedade acadêmica científica e profissionais da área da saúde. Assim, o objetivo deste estudo é avaliar os tipos de exercícios aeróbicos, em relação à frequência, intensidade e duração e os benefícios na redução do limiar da dor na fibromialgia.

\section{Metodologia}

O presente estudo trata-se de uma revisão sistemática (Soares et al., 2018) com abordagem quantitativa. Foram executadas as buscas de artigos datados entre 2010 a 2020 nas bases de dados SciELO, MEDLINE, PubMed, Periódicos CAPES e LILACS. As buscas por estudos foram realizadas no período de fevereiro a abril de 2020, por dois pesquisadores independentes, no entanto, por uma divergência o terceiro pesquisador foi utilizado. Inicialmente foram selecionados estudos que os títulos contemplassem os critérios de elegibilidade, posteriormente foram lidos os resumos, após a leitura dos resumos foi feita a leitura dos estudos na íntegra.

Na seleção dos artigos foram utilizados os descritores Fibromialgia, Dor, Exercício, Natação, Hidroterapia, Teste de Esforço, Dança, Protocolos, Caminhada e Treino Aeróbico utilizando o operador AND, seguindo as combinações entre os descritores nos idiomas inglês e português formando as strings de busca de acordo com o quadro abaixo.

Quadro 1. Strings de Busca da presente pesquisa.

\begin{tabular}{|l|l|l|l|}
\hline Fibromialgia AND Dor & Fibromialgia AND Exercício & $\begin{array}{l}\text { Fibromialgia AND } \\
\text { Exercício AND Dor }\end{array}$ & Fibromialgia AND Natação \\
\hline $\begin{array}{l}\text { Fibromialgia AND } \\
\text { Hidroterapia }\end{array}$ & $\begin{array}{l}\text { Fibromialgia AND Teste de } \\
\text { Esforço }\end{array}$ & Fibromialgia AND Dança & $\begin{array}{l}\text { Fibromialgia AND } \\
\text { Protocolos }\end{array}$ \\
\hline $\begin{array}{l}\text { Fibromialgia AND } \\
\text { Caminhada }\end{array}$ & $\begin{array}{l}\text { Fibromialgia AND Treino } \\
\text { Aeróbico }\end{array}$ & & - \\
\hline
\end{tabular}

Fonte: Autores.

Como critério de elegibilidade para a inclusão do estudo foram incluídos artigos que sejam relacionados ao público feminino, na faixa etária de 24 a 65 anos, ensaios clínicos (controlado/randomizado), estudos que usem a "dor" como desfecho primário, que utilizem como intervenção o exercício aeróbio. E como exclusão, artigos duplicados, e aqueles que apresentarem baixa qualidade metodológica após análise através da escala PEDro (Verhagen et al., 1998) com pontuação <5.

Após a seleção da análise metodológica os artigos foram analisados e apresentados através de fluxograma na Figura 1 e posteriormente discutidos. A pesquisa foi norteada pelos requisitos da escala PRISMA e utilizando o fluxograma Prisma (Moher et al., 2009). 
Figura 1 - Fluxograma de seleção dos artigos da revisão sistemática.

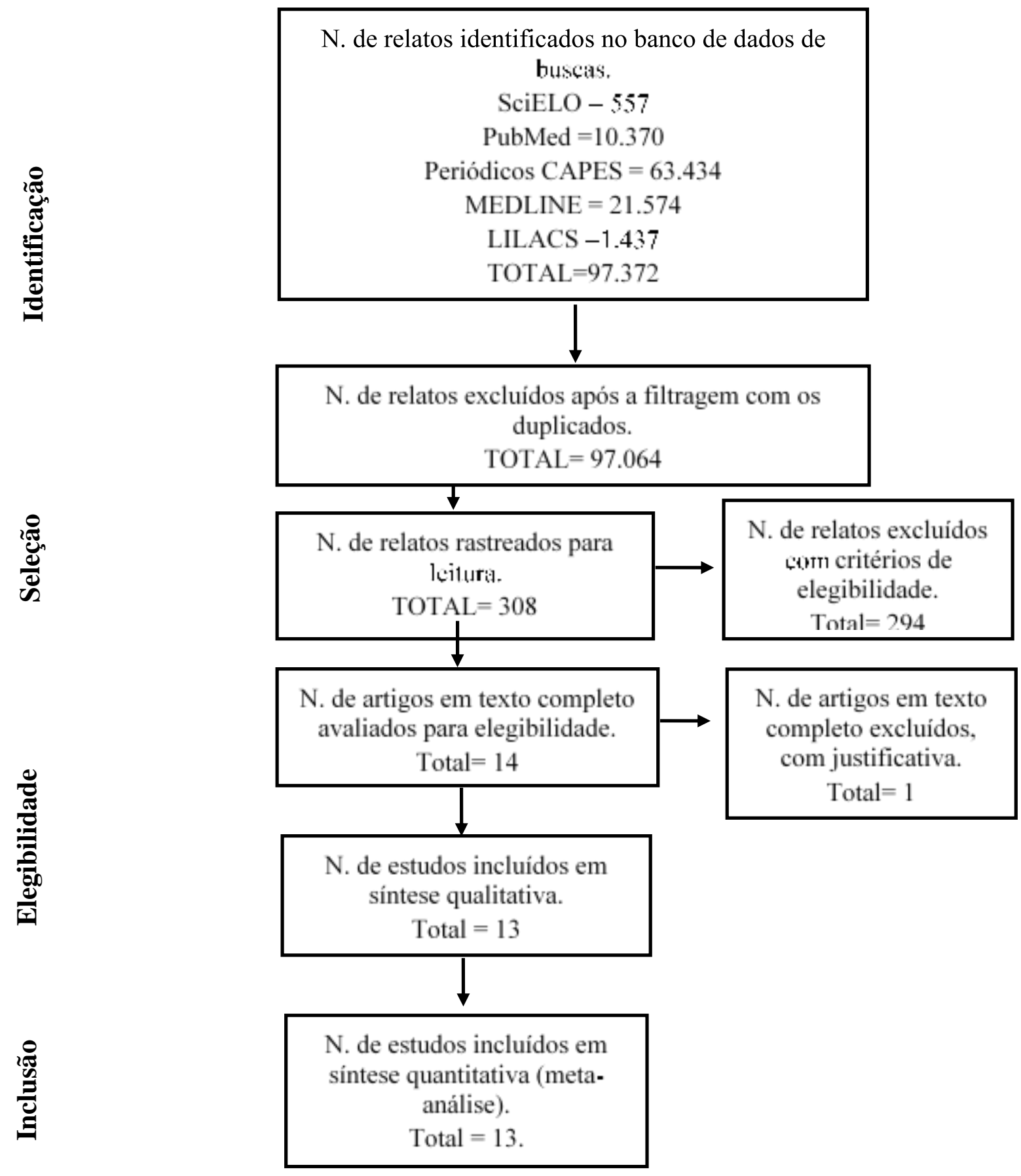

Fonte: Autores

\section{Resultados e Discussão}

Com base na pesquisa é possível afirmar que durante o tratamento de interversão de EA em relação a dor em paciente com FM, os estudos se mostram benéficos na abordagem de protocolos. Alguns estudos demonstram que certos sintomas como dores musculares generalizadas e pontos dolorosos específicos estão presentes em pessoas com a síndrome. Os resultados encontrados a nível de dor foram de forma positiva sobre a intervenção de EA como caminhada, Hidroterapia, Biodança e 
Bicicleta ergométrica em relação a tipos de intervenção de Exercício de Fortalecimento, Utilização de Tens e tai chi. Após a seleção da análise metodológica os artigos foram analisados e serão apresentados através de fluxograma (Figura 1) e posteriormente discutidos. Durante a pesquisa inicial nas bases de dados foi encontrado um total de 97.372 artigos com todas as combinações utilizadas.

Conforme utilizou-se a filtragem e retirado a duplicidade, foram excluídos 97.064 artigos, restando 308 artigos para revisão de títulos, resumo, e leitura na íntegra. Foi efetuado a exclusão de 294 pois os mesmos não cumpriram com os critérios de elegibilidade e após essa análise final, foram selecionados 14 artigos, sendo avaliados metodologicamente pela escala PEDro (Verhagen et al., 1998). Apenas um artigo (Zamunér et al., 2015) foi excluído por apresentar a pontuação menor que cinco na escala.

Quadro 2 - Qualidade metodológica dos artigos selecionados que foram incluídos e excluídos nesta revisão, segundo a escala PEDro.

\begin{tabular}{|c|c|c|c|c|c|c|c|c|c|c|c|}
\hline Autor/Ano & \multicolumn{10}{c|}{ Critérios } \\
\hline Borja Sañudo, 2010 & $\mathrm{X}$ & 2 & 3 & 4 & 5 & 6 & 7 & 8 & 9 & 10 & 11 \\
\hline Carbonell-Baeza, 2010 & $\mathrm{X}$ & - & - & $\mathrm{X}$ & - & - & - & $\mathrm{X}$ & $\mathrm{X}$ & $\mathrm{X}$ & $\mathrm{X}$ \\
\hline Kayo AH,2011 & $\mathrm{X}$ & $\mathrm{X}$ & $\mathrm{X}$ & $\mathrm{X}$ & - & - & $\mathrm{X}$ & $\mathrm{X}$ & $\mathrm{X}$ & $\mathrm{X}$ & $\mathrm{X}$ \\
\hline A.S. Baptista, 2012 & $\mathrm{X}$ & $\mathrm{X}$ & $\mathrm{X}$ & $\mathrm{X}$ & $\mathrm{X}$ & $\mathrm{X}$ & - & $\mathrm{X}$ & $\mathrm{X}$ & $\mathrm{X}$ & $\mathrm{X}$ \\
\hline B. Mutlu, 2012 & $\mathrm{X}$ & $\mathrm{X}$ & $\mathrm{X}$ & $\mathrm{X}$ & $\mathrm{X}$ & - & $\mathrm{X}$ & $\mathrm{X}$ & $\mathrm{X}$ & $\mathrm{X}$ & $\mathrm{X}$ \\
\hline Hooten WM. 2012 & $\mathrm{X}$ & $\mathrm{X}$ & $\mathrm{X}$ & $\mathrm{X}$ & - & - & - & $\mathrm{X}$ & $\mathrm{X}$ & $\mathrm{X}$ & $\mathrm{X}$ \\
\hline López Rodriguez MM, 2012 & $\mathrm{X}$ & - & - & $\mathrm{X}$ & - & - & - & $\mathrm{X}$ & - & $\mathrm{X}$ & $\mathrm{X}$ \\
& & & & & & & & & & & \\
\hline Lopez Rodriguez MM, 2012 & $\mathrm{X}$ & $\mathrm{X}$ & $\mathrm{X}$ & $\mathrm{X}$ & $\mathrm{X}$ & - & $\mathrm{X}$ & - & $\mathrm{X}$ & $\mathrm{X}$ & $\mathrm{X}$ \\
\hline Jiminez V, 2012 & & & & & & & & & & & \\
\hline Neslihan Duruturk, 2014 & $\mathrm{X}$ & - & - & - & - & - & - & $\mathrm{X}$ & $\mathrm{X}$ & $\mathrm{X}$ & $\mathrm{X}$ \\
\hline Ar Zamunir,2015 & $\mathrm{X}$ & - & - & $\mathrm{X}$ & $\mathrm{X}$ & - & - & $\mathrm{X}$ & $\mathrm{X}$ & $\mathrm{X}$ & $\mathrm{X}$ \\
\hline Fernandes G, 2016 & $\mathrm{X}$ & $\mathrm{X}$ & $\mathrm{X}$ & $\mathrm{X}$ & - & - & - & - & $\mathrm{X}$ & $\mathrm{X}$ & $\mathrm{X}$ \\
\hline Carolina P, 2018 & $\mathrm{X}$ & $\mathrm{X}$ & $\mathrm{X}$ & $\mathrm{X}$ & - & - & $\mathrm{X}$ & $\mathrm{X}$ & $\mathrm{X}$ & $\mathrm{X}$ & $\mathrm{X}$ \\
\hline Chenchen Wang, 2018 & $\mathrm{X}$ & $\mathrm{X}$ & $\mathrm{X}$ & $\mathrm{X}$ & - & - & - & $\mathrm{X}$ & $\mathrm{X}$ & $\mathrm{X}$ & $\mathrm{X}$ \\
\hline
\end{tabular}

Fonte: Autores.

Em análise, aos resultados obtidos nos 13 estudos especificamente selecionados, o quadro I contempla a análise da escala PEDro. Em seguida a Tabela 1 abrange respectivamente os resultados obtidos por colunas começando com as modalidades exercidas pelos estudos. Na segunda coluna estarão disponíveis a intensidade, em seguida a frequência, e depois na quarta coluna a duração. Na quinta coluna encontram-se os dados coletados sobre avaliação da dor, na sexta coluna está inclusa as amostras, e por fim o autor e ano de cada estudo. 
Tabela 1. Delineamento dos estudos, características das modalidades, Intensidade, Frequência, Duração, Escalas, Amostras e Autor/Ano.

\begin{tabular}{|c|c|c|c|c|c|c|}
\hline \multicolumn{7}{|l|}{ Modalidade } \\
\hline & Intensidade & Frequência & Duração & Dor & Amostra & Autor/Ano \\
\hline \multirow[t]{2}{*}{ Biodança } & $1 \mathrm{x}$ Por semana & $\begin{array}{l}\text { Controlada por Borg, Com } \\
\text { valor ERP } 11 \mathrm{AE} \text { de baixa } \\
\text { intensidade }\end{array}$ & 70 - 80 Min & $\begin{array}{c}\text { Algometria, Meter e } \\
\text { FIQ }\end{array}$ & 71 & $\begin{array}{l}\text { Ana Carbonell } \\
\text { - Baeza et al } \\
2010\end{array}$ \\
\hline & $\begin{array}{c}2 \times \text { Por } \\
\text { semana(total de } \\
16 \text { semanas) }\end{array}$ & - & 1 hora & $\begin{array}{l}\text { EVA, FIQ, BDI e SF- } \\
36\end{array}$ & 80 & $\begin{array}{l}\text { Baptista As et } \\
\text { al } 2012 .\end{array}$ \\
\hline \multirow[t]{2}{*}{ Caminhada } & 4 Semanas & $40-50 \%$ & $25-50$ Min & EVA,FIQ e SF-36 & 90 & $\begin{array}{l}\text { Kayo AH, Et } \\
\text { al } 2011\end{array}$ \\
\hline & 16 Semanas & $60-70 \%$ & 25-30 Min & EVA,FIQ e SF-36 & 90 & $\begin{array}{l}\text { Kayo AH, Et } \\
\quad \text { al } 2011\end{array}$ \\
\hline \multirow[t]{4}{*}{$\begin{array}{l}\text { Exercício } \\
\text { Aeróbico }\end{array}$} & $\begin{array}{c}2 \times \text { Por } \\
\text { semana(Total de } \\
\text { 24 Semanas) }\end{array}$ & $60 \%-65 \%$ & 45-60 Min & FIQ e BDI & 64 & $\begin{array}{l}\text { Borja Sañudo } \\
\text { et al } 2010\end{array}$ \\
\hline & $\begin{array}{c}2 \times \text { Por } \\
\text { semana(Total de } \\
24 \text { Semanas) }\end{array}$ & $75-80 \%$ & 45-60 Min & FIQ e BDI & 64 & $\begin{array}{l}\text { Borja Sañudo } \\
\text { et al } 2010\end{array}$ \\
\hline & $\begin{array}{c}1 \text { semana a } 9 \\
\text { Semana }\end{array}$ & $50-60 \%$ & 35 Min & FIQ e EVA & 226 & $\begin{array}{l}\text { ChenChen } \\
\text { Wang et al } \\
2018\end{array}$ \\
\hline & 10-12 Semanas & $60-70 \%$ & $40 \mathrm{Min}$ & FIQ e EVA & 226 & $\begin{array}{l}\text { ChenChen } \\
\text { Wang et al } \\
2018\end{array}$ \\
\hline \multirow[t]{2}{*}{ Ciclismo } & $\begin{array}{c}3 \times \text { Por } \\
\text { semana(Total de } \\
12 \text { semanas) }\end{array}$ & $60-70 \%$ & $40 \mathrm{Min}$ & $\begin{array}{l}\text { CPT,MPS,FIQ e } \\
\text { SDF-36 }\end{array}$ & 66 & $\begin{array}{l}\text { B.Mutlu et al } \\
2012\end{array}$ \\
\hline & Por Tres semana & $70-75 \%$ & 10-30 Min & Algometro & 72 & $\begin{array}{c}\text { Hooten WM et } \\
\text { al } 2012\end{array}$ \\
\hline \multirow[t]{2}{*}{$\begin{array}{l}\text { Biodança } \\
\text { Aquática }\end{array}$} & $\begin{array}{l}\text { 2x Por semana } \\
\text { (Total de } 12 \\
\text { semanas) }\end{array}$ & $\begin{array}{l}\text { temperatura da água de } \\
\text { aproximadamente } 29^{\circ} \mathrm{C}\end{array}$ & 1 hora & $\begin{array}{l}\text { FIQ, Questionário de } \\
\text { dor de Mcgill, EVA e } \\
\text { Algometria. }\end{array}$ & 82 & $\begin{array}{c}\text { López } \\
\text { Rodriguez } \\
\text { MM et al } 2012\end{array}$ \\
\hline & $\begin{array}{l}2 \text { Por semana( } 3 \\
\text { meses) }\end{array}$ & $\begin{array}{l}\text { temperatura da água de } \\
\text { aproximadamente } 29^{\circ} \mathrm{C}\end{array}$ & 1 Hora & $\begin{array}{c}\text { EVA, FIQ, } \\
\text { Questionario de } \\
\text { MCGill, EVA e } \\
\text { Algometria }\end{array}$ & 52 & $\begin{array}{c}\text { López } \\
\text { Rodriguez } \\
\text { MM et al } 2012\end{array}$ \\
\hline \multirow[t]{3}{*}{ EA Aquático } & $\begin{array}{c}\text { 2x Por } \\
\text { semana(Total de } \\
12 \text { Semanas) }\end{array}$ & $\begin{array}{l}\text { taxa de exercício percebido } \\
\text { (EPR) com base na escala } \\
\text { convencional de Borg. }\end{array}$ & 45 Min & Algometrica e EVA & 33 & $\begin{array}{l}\text { Segura- } \\
\text { Jiménez V et } \\
\text { al } 2012\end{array}$ \\
\hline & $\begin{array}{c}3 x \text { Por } \\
\text { semana(Total de } \\
12 \text { Semanas) }\end{array}$ & $\begin{array}{l}\text { FC Máxima de } 11 \text { batimentos } \\
\text { abaixo do limiar anaeróbico } \\
\text { (TA) e GP }\end{array}$ & 50 Min & EVA & 75 & $\begin{array}{l}\text { Fernandes G et } \\
\text { al } 2016\end{array}$ \\
\hline & $\begin{array}{l}\text { 2x Por semana( } \\
\text { Total de } 16 \\
\text { Semanas) }\end{array}$ & $\begin{array}{c}\text { Manter a FC e o esforço } \\
\text { subjetivo percebido atingido } \\
\text { no nível do VAT identificado } \\
\text { no CPET. }\end{array}$ & 45 Min & $\begin{array}{l}\text { OMERACT-10, } \\
\text { EVA, SF-36 e FIQ }\end{array}$ & 54 & $\begin{array}{l}\text { Carolina P. } \\
\text { Andrade et al } \\
\quad 2018 .\end{array}$ \\
\hline $\begin{array}{c}\text { Esteira } \\
\text { Ergométrica }\end{array}$ & $\begin{array}{l}3 \times \text { Por Semana( } \\
\text { Total de } 6 \\
\text { Semanas) }\end{array}$ & $60-75 \%$ & 20-45 Min & FIQ & 33 & $\begin{array}{c}\text { Neslihan } \\
\text { Duruturk et al } \\
2014\end{array}$ \\
\hline
\end{tabular}

Legendas: EA - Exercício Aeróbico. FIQ - Questionário de Impacto da Fibromialgia. BDI - Inventário de Depressão de Beck. FC máx. - Frequência Cardíaca Máxima Prevista. ERP- Taxa de Esforço Percebido. MPS - Escore de dor Mialgica. SF-36 - Inquérito de Saúde em Formato Curto. TCP - Contagem de Pontos de Concurso 0-18 pontos da Fibromialgia. CPET- Teste de esforço Cardiopulmonar. VAT- Limiar Anaeróbico Ventilatório. GP - Grupo de Caminhada. Fonte: Autores. 
A maioria das pesquisas de EA Aquático no qual foram abordados 3 estudos, seguindo por 2 Estudos pela Caminhada, 2 Biodança, 2 EA, Ciclismo, 2 Biodança Aquática e 1 Estudo de Caminhada e Esteira Ergométrica.

Quando verificado os 13 estudos 6 utilizaram as intensidades de $2 \mathrm{x}$ por semana no total de 12 semanas consecutivas, 1 estudo utilizou-se de 2 x por semana (Total de 3 meses) 1 estudo 3 x por Semanas (Total de 6 semanas) 2 estudos por 3x por semana (Total de 12 semanas), 5 dos estudos não tiveram Dias das semanas relatados, porém utilizaram de no mínimo 3 semanas consecutivas e 16 semanas.

Os Critérios de Frequência utilizado nos estudos avaliados foram 1 Estudo com 40-50\% Fc máxima,1 de 50-60\%, 3 de $60-70 \%, 1$ de $60-75 \% .1$ de 60-65,1 de 70-75\%, 1 de 75-80\%, 2 Mantiveram a temperatura da água de aproximadamente $29^{\circ} \mathrm{C}, 3$ FC Máxima de 11 batimentos abaixo do limiar anaeróbico (TA), GP e Escala de Borg. 1 pelo nível de Manter a Fc. Máxima e o esforço subjetivo percebido no Nível do VAT e identificado pelo teste de esforço cardiopulmonar (CPET), O estudo de Baptista et al (2012) não relatou a frequência utilizada.

Dentre os diversos estudos analisados, 12 Estudos mantiveram a duração de no mínimo 10 Min e máximo 1 hora, já o estudo de Wang et al (2018), teve duração de 70-80 min. Nas escalas utilizadas foram combinadas nas avaliações por 10 Estudos utilizaram a EVA, 13 (FIQ), 5 (SF-36), 3 (Algometria), 1 (Omeroct-10), 2 (MCGill), 1 (Meter), CPT, MPS e DBI.

As amostras coletadas nos estudos avaliados foram entre 33 e 226 pessoas que Somatizar um total de 998 amostras.

Analisando os Resultados dos Presentes estudos, indicam menos impacto negativos, melhorias significativas, reduzindo a gravidade da dor que chegam a ser reduzida de 14-15\% até 40\%. Diminuição de Trigger Points, consequentemente melhoram o quadro de ansiedade e depressão adquirida pela FM. Melhorando assim a qualidade de vida desses pacientes.

Comparando assim com o EA mesmo em diversas modalidades analisadas mostra eficácia no tratamento não medicamentoso prescrito, melhorando a $\mathrm{Vo}^{2}$, comprovando assim já a eficácia fisiológica cujo foi comprovada por uma pesquisa anterior. Os resultados benéficos da água fazem com que indivíduos tenham resultados positivos em relação a intensidade da dor.

A intervenção de no mínimo 4 semanas consecutivas no curto prazo, é um recurso muito útil para o gerenciamento da FM, sendo um programa de flexibilidade e reabilitação para as mulheres com SFM, porém se realizado um destreinamento nas manifestações clínicas revela que existe o retorno à linha base da Dor.

O presente estudo traz como base avaliar os protocolos de exercícios aeróbicos realizados em mulheres com fibromialgia. Foi observado que entre os diferentes programas de exercícios aeróbicos utilizados nos 13 estudos selecionados, que as atividades aeróbicas são utilizadas em todas as intervenções, e dentre os métodos utilizados encontram-se as atividades de caminhadas ao ar livre, bicicletas ergométricas, natação e danças. Desse modo, se correlacionando com os métodos utilizados na pesquisa.

Os protocolos que obtiveram melhor eficácia sobre os exercícios aeróbicos foram os que tiveram como intervenção correlacionar a frequência em torno do número de semanas consecutivas, no qual foi observado que há uma melhora no limiar da dor, nos quais começa a se mostrar eficaz a partir da 4 semana, porém, muitos dos pacientes acabam por desistir do tratamento antes das 4 semanas por uma diminuição no limiar da dor. Os pacientes que progridem com o tratamento e passam das 4 semanas tem uma redução significativa sobre o limiar da dor pelo qual se obtém uma redução e melhores resultados sobre a sintomatologia dolorosa.

Desse modo, observou-se que no protocolo utilizado por Baptista et al (2012), que oferece um exercício aeróbico (EA) como a dança do ventre, com duração de uma hora, sendo realizada duas vezes por semana num total de 16 semanas. Foram realizados exercícios de aquecimentos, pelo movimento determinado compromisso do dia, coreografia, e em sequência um exercício de relaxamento. E obtiveram uma eficácia relevante ao tratamento de SFM, pois, obteve $40 \%$ de redução da dor e que se estendeu durante 32 semanas. Os dados foram obtidos através de escala visual analógica EVA ( $p<0,001$ ) e por meio da 
FIQ ( $\mathrm{p}=0,003)$ que é um instrumento que mede diversos aspectos da FM e seus impactos sobre o indivíduo afetado. Além de ter sido observado através do teste de caminhada de seis minutos $(\mathrm{p}<0,001)$ que os pacientes apresentaram melhorias na capacidade funcional desses indivíduos.

Corroborando com o protocolo anterior um estudo realizado por Wang et al (2018), comparou o tai chi e exercício aeróbico (coreografado), administrado com a mesma intensidade e duração por 12 ou 24 semanas, duas vezes por semana em dois grupos designados aleatoriamente para EA por 24 semanas ou um dos quatro estilos do yang clássico do tai chi por 12 ou 24 semanas, uma ou duas vezes semanal. O tratamento de tai chi comparado ao EA com a mesma intensidade e duração de 24 semanas, duas vezes por semana, obteve maior benefício quando comparado ao grupo que fez uso do tai chi por 12 semanas dentro do escore de questionário do impacto da fibromialgia (FIQR). Desse modo, além de resultar em maiores benefícios nos sintomas da FM do que os EA, o tai chi permite uma abordagem mente-corpo que resulta em melhorias nos sintomas da FM podendo vir a colaborar como tratamento não medicamentoso, independente de dosagem ou duração. Tornando-se uma opção terapêutica desses indivíduos, podendo proporcionar uma maior adesão pelas pessoas e maior manejo no tratamento multidisciplinar da fibromialgia. Em relação ao FIQR houve uma diferença entre grupos $=5,5$ pontos, intervalo de confiança de $95 \%$ de 0,6 a $10,4, P=0,03)$.

No estudo realizado por Mutlu et al (2013), investigou o uso do exercício supervisionado com eletrocardiograma transcutâneo e estimulação do nervo tricíclico (TENS). A intervenção foi realizada em dois grupos de tratamento. Todos os pacientes participaram de um acompanhamento supervisionado de 40 minutos, programa de exercícios combinados em 3 dias por semana durante 12 semanas. A melhoria do MPS na terceira semana foi maior no primeiro grupo $(p=0,01)$. A diminuição do MPS não apresentou diferença estatisticamente significante na $12^{\circ}$ semana entre os grupos $(p=0,87)$. Não houve diferença significativa entre a melhoria nos outros parâmetros de resultado dos dois grupos. Desse modo, foi observado que o programa de exercícios supervisionados teve sucesso em melhorar a dor miálgica. Além de combinar exercícios com TENS que podem ser úteis devido ao alívio rápido da dor miálgica no tratamento da FM na prática cotidiana e na melhora da qualidade de vida de mulheres portadoras da SFM.

O protocolo sugerido por Andrade et al (2019), mostra a eficácia sobre o treinamento físico aquático pelo qual os efeitos da água se manifestam, e reduzem a sintomatologia dolorosa em mulheres com FM. O programa foi realizado em uma piscina aquecida $\left(30^{\circ} \mathrm{C} \pm 2{ }^{\circ} \mathrm{C}\right)$. O protocolo consistiu em 32 sessões de $45 \mathrm{~min}$, duas vezes por semana (dias alternados) por 16 semanas. O presente estudo se mostrou eficaz até 16 semanas onde foi possível promover um aumento do VO2 e aumento do IVA e pico de CPTE melhorando assim, a sintomatologia da FMS em mulheres. De acordo com os critérios de resposta do OMERACT-10 FM, os pacientes apresentaram 7\% de redução da dor na EVA, aumento de 192\% na função física do SF-36 e redução de 19\% no FIQ, após 16 semanas de APT. Em contrapartida, após o período de destreinamento, o grupo treinado apresentou redução do PPT ( $p<0,01)$ e bem-estar ( $p=0,01$ ), e um aumento no FIQ ( $p<0,01)$ e dor na EVA ( $p=0,02)$. Assim, a APT deve ser realizada continuamente, a fim de melhorar a qualidade clínica, sintomatologia e aumentar a capacidade funcional aeróbica de mulheres com SFM.

Já o estudo de Segura-Jiménez et al (2013) mostrou os benefícios imediatos da dor, com um programa de intervenção na piscina que ocorreu durante 12 semanas e em 2 sessões, com duração de 45 minutos. A intensidade do treinamento foi controlada pela taxa de exercício percebido (EPR) com base na escala convencional de Borg. Não houve diferenças significativas nas características dos sujeitos da linha (idade, EVA, CPT e IMC) dos grupos de exercícios foram encontrados (todos $p>0,05$ ). Foram observados os benefícios de uma redução de $15 \%$ da intensidade imediata da dor na comparação da EVA, valores antes e depois de cada sessão com uma média decrescente de 1,04 $\pm 0,06$. Sem diferenças significativas (valor $\mathrm{P}$ de 0,51 a 1,0$)$ entre a amostra final.

Assim como no estudo realizado por Fernandes et al (2016), que avaliou os exercícios aeróbicos e os relacionou à 
natação em paciente com FM, o mesmo evidenciou mudanças nos grupos que se submeteram ao protocolo adotado que utilizou água como recurso para os exercícios. Os participantes foram avaliados antes dos protocolos de exercício (T0), em seis semanas (T6) e às 12 (T12) semanas após o início dos protocolos. Como método primário foi utilizado a (EVA) para avaliar o nível de dor desses pacientes. E como método secundário a (FIQR) e Medical Outcome Survey Short Form 36 (SF-36) para avaliar a qualidade de vida desses pacientes. Os pacientes de ambos os grupos apresentaram melhora na dor após as 12 semanas do programa, porém sem diferença entre os grupos ( $\mathrm{p}=0,658)$. Ambos os grupos apresentaram redução semelhante no uso de analgésicos durante todo o período experimental. Esse achado sugere que a redução da dor foi resultado dos programas de exercícios. Além disso, a alta frequência de atendimento em ambos os grupos (77,9\% no GE e 72,8\% no GT) demonstram que o protocolo do meio aquático tem aderência semelhante à do protocolo de caminhada.

Em respeito ao estudo realizado por Carbonell-Baeza et al (2010), que avaliou os efeitos de uma intervenção por meio da biodança, e constatou que durante o período do estudo houveram mudanças na redução e no impacto da FM em pacientes. A intervenção por meio da biodança ocorreu 1 vez por semana, durante 3 meses. Cada sessão durou 120 minutos e foi dividida em duas partes: a primeira fase verbal de 35 a 45 minutos. A segunda seria a própria vivência (experiência de 75 a 80 minutos), que envolve mover / dançar de acordo com a sugestão dada pelo facilitador e pela música tocada. Obtiveram como resultado uma melhora significativa na pontuação do algômetro ( $p<0,008$ ), diminuição na contagem de pontos sensíveis ( $p<0,002)$ e pontuação total do FIQ ( $p<0,003)$. Os dados obtidos mostraram que a intervenção por meio da biodança é um recurso de grande utilidade para o gerenciamento da FM, tendo em vista que há redução na dor ocasionada por essa SFM.

Como no estudo realizado por López-Rodríguez et al (2012), que utilizou a biodança aquática como método terapêutico, O protocolo foi utilizado 1 vez por semana com duração de 1 hora, por um período de 3 meses (12 semanas), se mostrou com benéfico na melhoria da dor e redução dos pontos dolorosos na SFM. Após o tratamento, observaram diferenças significativas no grupo experimental ( $\mathrm{P}<0,05)$, na qualidade do sono $(49,7 \%)$, ansiedade $(14,1 \%)$, impacto da fibromialgia (18,3\%), dor (27,9\%), McGill (23,7\%) e pontos de dolorosos (34,4\%). O que nos mostra que o protocolo adotado ao longo dos 3 meses de adesão, trouxe melhorias na qualidade do sono, estado de ansiedade, dor e outros sintomas da fibromialgia, produzindo uma melhora na qualidade de vida desses pacientes.

Em outro estudo realizado por López-Rodríguez et al (2013) , é avaliado o nível de melhora em relação à dor. O grupo de biodança realizou o tratamento em piscina, com duração de 1 hora para cada sessão. Essas sessões foram realizadas duas vezes por semana, durante um período de 12 semanas, cada uma dividida em 3 partes. Após a intervenção, analisando as diferenças intragrupo, o grupo experimental obteve uma redução significativa no escore FIQ (IC 95\%: -23,50 a -5,39; p <0,04), do questionário de dor de McGill. (IC95\%: -14,50 a -7, p <0,01) e EVA (IC95\%: -2,50 a 0, p <0,02), bem como na algometria em todos pontos de concurso. No GC, por outro lado, não foram encontradas diferenças significativas entre os grupos para nenhuma variável, exceto para a algometria de alguns pontos. E por fim, no inventário de depressão de Beck, não foram encontradas diferenças significativas entre os grupos. Desse modo, como preconizado nos estudos anteriores a biodança solo e biodança aquática assemelham-se aos resultados obtidos nesses estudos, os métodos adotados mostraram-se benéficos na redução da sintomatologia dolorosa dos portadores da SFM.

Em respeito ao protocolo utilizado por Duruturk et al (2015), que comparou os efeitos de treinamento de equilíbrio em balança ao treinamento de exercícios aeróbicos correlacionando-os com os sinais e sintomas da fibromialgia, tendo encontrado semelhança entre eles. Os exercícios foram realizados três vezes por semana, durante 6 semanas em esteira ou com um sistema de balança interativa Tetrax (TIBS). O resultado e medidas foram caracterizados por escore mialgico, questionário de impacto da fibromialgia (FIQ), exercício medição de tensão, medição Timed Up-Go (TUG) e TIBS. Diferenças significativas foram encontradas para intensidade geral da dor, intensidade da dor em repouso e atividade e escore miálgica total no grupo EA (p $<0,05$ ). Houve melhorias significativas nos valores do FIQ no grupo EA ( $p<0,05)$. No entanto, os pacientes, randomizados 
para o grupo EA, apresentaram maiores efeitos sobre a dor e a função física, que são as diversas medidas de resultados deste estudo e alcançaram maiores ganhos nesses resultados em comparação com os pacientes randomizados para o grupo BE.

Segundo Kayo et al (2012), comparando assim o protocolo na rotina de exercício aeróbico sobre a caminhada em grupos randomizados, onde comparou os efeitos da força e o EA revelaram equivalência em relação a melhora da dor, reduzindo dessa forma sua severidade. Os pacientes também foram examinados após 8 (T8) e 16 (T16) semanas de tratamento e 12 semanas após o final do estudo protocolo (28 semanas de seguimento, T28). Portanto, os dados foram coletados em 4 momentos: T0, T8, T16 e T28 para um total de 7 meses de acompanhamento (incluindo o período de tratamento). A redução da dor foi semelhante nos grupos de caminhada e fortalecimento muscular $(\mathrm{P}=0,39)$, mas diferente do grupo controle $(\mathrm{P}=$ 0,01). Os escores totais médios do FIQ foram menores nos grupos de caminhada e fortalecimento muscular $(\mathrm{P}=0,96) \mathrm{em}$ comparação com o grupo controle $(\mathrm{P}<0,01)$. Com a similaridade entre os métodos utilizados, não há um consenso em qual obteve melhores resultados, pois ambos os resultados revelaram que ambas as modalidades de exercício proporcionaram melhor alívio da dor em pacientes com fibromialgia.

Desse modo um estudo realizado por Hooten et al (2012), corrobora que tanto o EA quanto os exercícios de fortalecimento muscular, são abrangentes em relação ao alívio da severidade da dor e apresentem similaridade na redução dos sintomas da FM, ambos propiciaram melhorias significativas no aspecto fisiológico, condicionamento aeróbico e dor por pressões limiares. As admissões ao programa ocorrem de forma rotativa e os pacientes atendem $8 \mathrm{~h}$ diariamente por 15 dias úteis consecutivos, ou seja, 3 semanas. A intervenção do condicionamento aeróbico incluiu o uso de uma bicicleta ary. Melhorias significativas foram observadas na gravidade da dor ( $\mathrm{P}<0,001)$, pico de $\mathrm{Vo}$ (2) ( $\mathrm{P}<0,001)$, força ( $\mathrm{P}<0,001)$ e limiares de dor $(\mathrm{P}<0,001)$ foram observadas desde a linha de base até a terceira semana, no entanto, os pacientes no grupo aeróbio (variação média de 2,0 $\pm 2,6 \mathrm{~mL} / \mathrm{kg} / \mathrm{min}$ ) apresentaram ( $\mathrm{P}<0,013$ ) no pico de Vo (2) em comparação ao grupo de força (variação média de $0,4 \pm 2,6 \mathrm{~mL} / \mathrm{kg} / \mathrm{min}$ ). Apesar de sua similaridade quando relacionado a dor, o grupo de EA obteve ganhos com relação ao VO2 comparados ao grupo de força.

Assim como no estudo realizado por Sanudo et al (2010), que optou por um programa combinado de exercícios aeróbicos, exercícios de fortalecimento muscular e flexibilidade. Os participantes foram alocados em 1 dos 3 grupos: EA supervisionado, CE supervisionado ou controle de cuidados habituais. As sessões de exercícios foram realizadas duas vezes por semana (45-60min /sessão) por 24 semanas. Uma melhoria de $14 \%$ a $15 \%$ em relação à linha de base no total do escore FIQ foi observado nos grupos de exercício (P. 02) e foi acompanhado por reduções nas pontuações do BDI de 8,5 (P .001) e 6.4 (P .001) pontos nos grupos $\mathrm{AE}$ e $\mathrm{CE}$, respectivamente ativamente. Em relação aos controles não exercitados, a $\mathrm{CE}$ provocou melhorias no SF-36 (P .003) e no corpo a dor (P .003) e foi mais eficaz que o EA provocando melhorias na Vitalidade (P .002) e Mental Domínios de saúde (P .04). Os dados obtidos pelos métodos citados corroboram entre si e sugerem que mulheres com SFM podem obter benefícios adicionais à saúde participando de um volume semelhante de CE. A principal medida de resultado foi o FIQ. As medidas de desfecho foram SF-36, BDI e TC6. No entanto, o estudo sugere que mulheres com SFM podem obter benefícios adicionais à saúde participando de um volume semelhante e um programa combinado de exercícios aeróbicos, exercícios de fortalecimento muscular e flexibilidade.

Os estudos evidenciados mostraram-se benéficos em sua grande maioria, e abrangem diversos protocolos relacionados à FM. Sabendo que fibromialgia é uma síndrome dolorosa e que se interliga diretamente ao esforço do indivíduo. Os protocolos mais predominantes entre os autores foram que as FC fossem de baixa à moderada de $40 \%$ a $80 \%$ não ultrapassando a zona de limiar anaeróbico, bem como o excitatório que poderiam vir a comprometer a eficácia do protocolo adotado, vindo a aumentar dessa forma o quadro álgico. 


\section{Conclusão}

Foram observadas melhorias estatisticamente significativas nos respectivos estudos citados, que incluem os aspectos relacionados à saúde física, redução da dor e, ainda, as percepções dos indivíduos relacionadas à FM. Muitos autores afirmam que os protocolos adotados durante o tratamento que utilizaram como recurso às terapias não medicamentosas são essenciais para que os indivíduos sejam encorajados ao tratamento contínuo e ininterrupto.

Relacionando os dados obtidos, os estudos sugerem que os diferentes protocolos adotados se mostraram benéficos na redução da sintomatologia dolorosa da FM, desde que, associam-se ao nível de dor em mulheres que tenham idade entre 24 e 65 anos. O presente estudo corrobora que os pacientes que aderirem aos protocolos supracitados terão ganhos significativos, desde que adotem como prescrição de protocolos as atividades de no mínimo 2x3 vezes por 4 semanas consecutivas de modo ininterruptos, com intensidade baixa de $40 \%$ podendo haver progressão para moderada, respeitando a adaptação do paciente e não ultrapassando $80 \%$ da sua FC. A sua duração deve ter início de 30 minutos a 1 hora a cada treinamento realizado, não ultrapassando a execução de 1 vez ao dia. Sugere-se novos estudos que possam abordar outras atividades e com um grupo mais seleto de mulheres.

\section{Referências}

American College of Sports Medicine. (1998). A quantidade e o tipo recomendados de exercícios para o desenvolvimento e a manutenção da aptidão cardiorrespiratória e muscular em adultos saudáveis. Revista Brasileira de Medicina Do Esporte, 4(3), 96-106. 10.1590/s1517-86921998000300005

Andrade, C. P., Zamunér, A. R., Forti, M., Tamburús, N. Y., \& Silva, E. (2019). Effects of aquatic training and detraining on women with fibromyalgia: Controlled randomized clinical trial. European Journal of Physical and Rehabilitation Medicine, 55(1), 79-88. 10.23736/S1973-9087.18.05041-4

Assunção Júnior, J. C., de Almeida Silva, H. J., da Silva, J. F. C., da Silva Cruz, R., de Almeida Lins, C. A., \& de Souza, M. C. (2018). Zumba dancing can improve the pain and functional capacity in women with fibromyalgia. Journal of Bodywork and Movement Therapies, 22(2), 455-459. 10.1016/j.jbmt.2017.09.022

Baptista, A. S., Villela, A. L., Jones, A., \& Natour, J. (2012). Effectiveness of dance in patients with fibromyalgia: a randomized, single-blind, controlled study . Clinical and Experimental Rheumatology, 30((6 Suppl 74)), 18-23. https://pubmed.ncbi.nlm.nih.gov/23020850/

Batista, E. D., Andretta, A., de Miranda, R. C., Nehring, J., Paiva, E. dos S., \& Schieferdecker, M. E. M. (2016). Food intake assessment and quality of life in women with fibromyalgia. Revista Brasileira de Reumatologia, 56(2), 105-110. 10.1016/j.rbre.2015.08.015

Boter, D. F., \& Testa Junior, L. P. N. A. (2020). Adaptações cardiovasculares subsequententes aos exercícios físicos aeróbios ou resistidos | Revista MotriSaúde. Revista MotriSaúde, 2(1). http://revista.fundacaojau.edu.br:8078/journal/index.php/revista_motrisaude/article/view/198

Carbonell-Baeza, A., Aparicio, V. A., Martins-Pereira, C. M., Gatto-Cardia, C. M., Ortega, F. B., Huertas, F. J., Tercedor, P., Ruiz, J. R., \& DelgadoFernandez, M. (2010). Efficacy of biodanza for treating women with fibromyalgia. Journal of Alternative and Complementary Medicine, 16(11), 1191-1200. $10.1089 / \mathrm{acm} .2010 .0039$

Carvalho, L. S. C., Correa, H., Silva, G. C., Campos, F. S., Baião, F. R., Ribeiro, L. S., Faria, A. M., \& D’Avila Reis, D. (2008a). May genetic factors in fibromyalgia help to identify patients with differentially altered frequencies of immune cells? Clinical \& Experimental Immunology, 154(3), 346-352. 10.1111/j.1365-2249.2008.03787.x

Clark, P., Gentile, M. J., Helfenstein, M., Jannaut, M. J., Liendo, V., Ríos, C., Vidal Neira, L., \& Messina, O. D. (2011). Diagnóstico y tratamiento farmacológico y no farmacológico de la fibromialgia. Síntesis de la mejor evidencia. In Drugs of Today (Vol. 47, Issue SUPPL. A, pp. 1-28). Drugs Today (Barc). 10.1358/dot.2011.471619953

Consenza, P. I. C. (2000). nfluência do volume de uma atividade aeróbica de intensidade moderada no desempenho subseqüente de in the Treatment of Hypertension: An Update. Medicine \& Science in Sports \& Exercise, 30(3), 193-206.

Cooper, H. K. (1982). O Programa Aerobico para o Bem-estar Total. Nordica.

Crofford, L. J., Young, E. A., Engleberg, N. C., Korszun, A., Brucksch, C. B., McClure, L. A., Brown, M. B., \& Demitrack, M. A. (2004). Basal circadian and pulsatile ACTH and cortisol secretion in patients with fibromyalgia and/or chronic fatigue syndrome. Brain, Behavior, and Immunity, 18(4), 314-325. 10.1016/j.bbi.2003.12.011

Cruz, R. da S., Assunção Júnior, J. C., Silva, H. J. de A., \& Souza, M. C. de. (2017). Experience with women with fbromyalgia who practice zumba. Case reports. Revista Dor, 18(3), 266-269. 10.5935/1806-0013.20170113

del Vecchio, F., Galliano, L., \& Coswig, V. (2013). Aplicações do exercício intermitente de alta intensidade na síndrome metabólica. Revista Brasileira de Atividade Física \& Saúde, 18(06), 669-669. 10.12820/rbafs.v.18n6p669

Duruturk, N., Tuzun, E. H., \& Culhaoglu, B. (2015). Is balance exercise training as effective as aerobic exercise training in fibromyalgia syndrome? Rheumatology International, 35(5), 845-854. 10.1007/s00296-014-3159-z 
Fernandes, G., Jennings, F., Nery Cabral, M. V., Pirozzi Buosi, A. L., \& Natour, J. (2016). Swimming Improves Pain and Functional Capacity of Patients With Fibromyalgia: A Randomized Controlled Trial. Archives of Physical Medicine and Rehabilitation, 97(8), 1269-1275. 10.1016/j.apmr.2016.01.026

Fitzcharles, M. A., Ste-Marie, P. A., Goldenberg, D. L., Pereira, J. X., Abbey, S., Choinière, M., Ko, G., Moulin, D. E., Panopalis, P., Proulx, J., \& Shir, Y. (2013). 2012 Canadian guidelines for the diagnosis and management of fibromyalgia syndrome: Executive summary. In Pain Research and Management (Vol. 18, Issue 3, pp. 119-126). Hindawi Limited. 10.1155/2013/918216

Häuser, W., Thieme, K., \& Turk, D. C. (2010). Guidelines on the management of fibromyalgia syndrome - A systematic review. In European Journal of Pain. 14(1), 5-10. 10.1016/j.ejpain.2009.01.006

Helfenstein, M., Goldenfum, M. A., \& Siena, C. A. F. (2012). Fibromyalgia: Clinical and occupational aspects. In Revista da Associacao Medica Brasileira (Vol. 58, Issue 3, pp. 358-365). Elsevier Editora Ltda. 10.1590/S0104-42302012000300018

Heymann, R. E., Paiva, E. D. S., Helfenstein, M., Pollak, D. F., Martinez, J. E., Provenza, J. R., Paula, A. P., Althoff, A. C., E Souza, E. J. D. R., Neubarth, F., Lage, L. V., Rezende, M. C., de Assis, M. R., Lopes, M. L. L., Jennings, F., da Costa Araújo, R. L. C., Cristo, V. V., Costa, E. D. G., Kaziyama, H. H. S., ... Fonseca, M. C. M. (2010). Consenso brasileiro do tratamento da fibromialgia. Revista Brasileira de Reumatologia, 50(1), 56-66. 10.1590/S048250042010000100006

Hooten, W. M., Qu, W., Townsend, C. O., \& Judd, J. W. (2012). Effects of strength vs aerobic exercise on pain severity in adults with fibromyalgia: A randomized equivalence trial. Pain, 153(4), 915-923. 10.1016/j.pain.2012.01.020

Kayo, A. H., Peccin, M. S., Sanches, C. M., \& Trevisani, V. F. M. (2012). Effectiveness of physical activity in reducing pain in patients with fibromyalgia: A blinded randomized clinical trial. Rheumatology International, 32(8), 2285-2292. 10.1007/s00296-011-1958-Z

Klumb, A., Steffens, R. D. A. K., do Vale, B. R., Fonseca, A. B. P., Da, M., Viana, S., Brandt, R., \& Andrade, A. (2012). Effect of practical of walk on the quality life and self-efficacy of women with fibromyalgia syndrome. In Revista Brasileira de Ciência e Movimento. 20(1). https://portalrevistas.ucb.br/index.php/RBCM/article/view/1894

Ljubojević, A., Jakovljević, V., \& Popržen, M. (2014). Effects Of Zumba Fitness Program On Body Composition Of Women. 10(1), 29-33. 10.5550/sgia.141001.en.004L

López-Rodríguez, M. D. M., Castro-Sánchez, A. M., Fernández-Martínez, M., Matarán-Peñarrocha, G. A., \& Rodríguez-Ferrer, M. E. (2012). Comparación entre biodanza en medio acuático y stretching en la mejora de la calidad de vida y dolor en los pacientes con fibromialgia. Atencion Primaria, 44(11), 641650. 10.1016/j.aprim.2012.03.002

Lorena, S. B. de, Pimentel, E. A. dos S., Fernandes, V. M., Pedrosa, M. B., Ranzolin, A., \& Duarte, A. L. B. P. (2016). Evaluation of pain and quality of life of fibromyalgia patients. Revista Dor, 17(1), 8-11. 10.5935/1806-0013.20160003

Mandini, M.-J., \& Michel, J.-P. (2001). Atividade física para adultos com mais de 55 anos: Quadros Clínicos E Programas De Exercícios | Amazon.com.br (1st ed., Vol. 1). Editora Manole.

Mannerkorpi, K. (2005). Exercise in fibromyalgia. In Current Opinion in Rheumatology 17(2), 190-194. Curr Opin Rheumatol. 10.1097/01.bor.0000154202.56962.85

Marques, Amélia Pasqual, Matsutani, L. A., Alves, E., Ferreira, G., Laís, E., Furtado De Mendonça, L., \& de Revisão, A. (2002). Physical therapy in the treatment of patients with fibromyalgia: a literature review. Rev Bras Reumatol-, 42, 42-48.

Marques, Amelia Pasqual, Santo, A. de S. do E., Berssaneti, A. A., Matsutani, L. A., \& Yuan, S. L. K. (2017). Prevalence of fibromyalgia: literature review update. Revista Brasileira de Reumatologia (English Edition), 57(4), 356-363. 10.1016/j.rbre.2017.01.005

Martins, M. R. I., Gritti, C. C., Junior, R. dos S., Araújo, M. C. L. de, Dias, L. C., Foss, M. H. D. A., Andrade, L. B. de, \& Rocha, C. E. D. A. (2014). Estudo randomizado e controlado de uma intervenção terapêutica grupal em pacientes com síndrome fibromiálgica. Revista Brasileira de Reumatologia, 54(3), 179184. 10.1016/j.rbr.2013.10.005

Matsutani, L. A., Assumpção, A., \& Marques, A. P. (2012). Exercícios de alongamento muscular e aeróbico no tratamento da fibromialgia: estudo piloto. Fisioterapia Em Movimento, 25(2), 411-418. 10.1590/s0103-51502012000200019

Mcardle, W. (2016). Fisiologia do Exercício - Nutrição, Energia e Desempenho Humano (8th ed.). Guanabara Koogan.

Moher, D., Liberati, A., Tetzlaff, J., \& Altman, D. G. (2009). Preferred reporting items for systematic reviews and meta-analyses: The PRISMA statement. In BMJ (Online) (Vol. 339, Issue 7716, pp. 332-336). British Medical Journal Publishing Group. 10.1136/bmj.b2535

Mosmann, A., Antunes, C., de Oliveira, D., Lara, C., \& Neves, M. (2006). Atuação fisioterapêutica na qualidade de vida do paciente fibromiálgico Physiotherapeutic actions in the quality of life of patients with fibromyalgia. In Scientia Medica 16(4). https://revistaseletronicas.pucrs.br/ojs/index.php/scientiamedica/article/view/2284

Mutlu, B., Paker, N., Bugdayci, D., Tekdos, D., \& Kesiktas, N. (2013). Efficacy of supervised exercise combined with transcutaneous electrical nerve stimulation in women with fibromyalgia: A prospective controlled study. Rheumatology International, 33(3), 649-655. 10.1007/s00296-012-2390-8

Pereira Pernambuco, A. (2014). Impacto De Um Programa De Educação Em Saúde Sobre Aspectos Neuroimunocomportamentais De Pacientes Com Diagnóstico De Fibromialgia. Universidade Federal de Minas Gerais. https://repositorio.ufmg.br/handle/1843/BUOS-9N8H4B

Pollock, M. L. (1993). Exercícios na saúde e na doença: avaliação e prescrição para prevenção e reabilitação (2nd ed.). MEDSI.

Ribeiro, K. L., \& Marinho, I. de S. F. (2005). Fibromialgia e Exercício. Fitness \& Performance Journal, $280-287$. https://www.redalyc.org/pdf/751/75117015003.pdf 
Riberto, M., \& Pato, T. R. (2004). Fisiopatologia da fibromialgia. Acta Fisiátrica, 11(2), 78-81. 10.5935/0104-7795.20040002

Sabbag, L. M. dos S., Dourado, M. P., Júnior, P. Y., Novo, N. F., Kaziyama, H. H. S., Miyazaki, M. H., \& Battistella, L. R. (2000). Estudo ergométrico evolutivo de portadoras de fibromialgia primária em programa de treinamento cardiovascular supervisionado. In Acta Fisiátrica (Vol. 7, Issue 1). 10.11606/ISSN.2317-0190.V7I1A102253

Saudo, B., Galiano, D., Carrasco, L., Blagojevic, M., de Hoyo, M., \& Saxton, J. (2010). Aerobic exercise versus combined exercise therapy in women with fibromyalgia syndrome: A randomized controlled trial. Archives of Physical Medicine and Rehabilitation, 91(12), 1838-1843. 10.1016/j.apmr.2010.09.006

Segura-Jiménez, V., Carbonell-Baeza, A., Aparicio, V. A., Samos, B., Femia, P., Ruiz, J. R., \& Delgado-Fernández, M. (2013). A warm water pool-based exercise program decreases immediate pain in female fibromyalgia patients: Uncontrolled clinical trial. International Journal of Sports Medicine, 34(7), 600 605. $10.1055 / \mathrm{s}-0032-1329991$

Senna, E. R., de Barros, A. L. P., Silva, E. O., Costa, I. F., Pereira, L. V. B., Ciconelli, R. M., \& Ferraz, M. B. (2004). Prevalence of rheumatic diseases in Brazil: a study using the COPCORD approach. The Journal of Rheumatology, 31(3).

Soares, A., Dorlivete, P., Shitsuka, M., Parreira, F. J., \& Shitsuka, R. (2018). Metodologia da pesquisa científica Núcleo de Tecnologia Educacional da Universidade Federal de Santa Maria p.

Verhagen, A. P., de Vet, H. C. W., de Bie, R. A., Kessels, A. G. H., Boers, M., Bouter, L. M., \& Knipschild, P. G. (1998). The Delphi list: A criteria list for quality assessment of randomized clinical trials for conducting systematic reviews developed by Delphi consensus. Journal of Clinical Epidemiology, 51(12), 1235-1241. 10.1016/S0895-4356(98)00131-0

Wang, C., Schmid, C. H., Fielding, R. A., Harvey, W. F., Reid, K. F., Price, L. L., Driban, J. B., Kalish, R., Rones, R., \& McAlindon, T. (2018). Effect of tai chi versus aerobic exercise for fibromyalgia: Comparative effectiveness randomized controlled trial. BMJ (Online), 360. 10.1136/bmj.k851

Wolfe, F., Clauw, D. J., Fitzcharles, M. A., Goldenberg, D. L., Katz, R. S., Mease, P., Russell, A. S., Russell, I. J., Winfield, J. B., \& Yunus, M. B. (2010). The American College of Rheumatology preliminary diagnostic criteria for fibromyalgia and measurement of symptom severity. Arthritis Care and Research, 62(5), 600-610. 10.1002/acr.20140

Wolfe, F., Smythe, H. A., Yunus, M. B., Bennett, R. M., Bombardier, C., Goldenberg, D. L., Tugwell, P., Campbell, S. M., Abeles, M., Clark, P., Fam, A. G., Farber, S. J., Fiechtner, J. J., Michael Franklin, C., Gatter, R. A., Hamaty, D., Lessard, J., Lichtbroun, A. S., Masi, A. T., \& Sheon, R. P. (1990). The american college of rheumatology 1990 criteria for the classification of fibromyalgia. Arthritis \& Rheumatism, 33(2), 160-172. 10.1002/art.1780330203

Zamunér, A. R., Andrade, C. P., Forti, M., Marchi, A., Milan, J., Avila, M. A., Catai, A. M., Porta, A., \& Silva, E. (2015). Effects of a hydrotherapy programme on symbolic and complexity dynamics of heart rate variability and aerobic capacity in fibromyalgia patients. Clinical and Experimental Rheumatology, 33(88), S73-S81. https://europepmc.org/article/med/25786047 